\title{
Sensing Machines
}

\section{The Ride}

I still remember the train ride, after a long flight, off to the next destination, soon to arrive. I don't often take the Inter-City Express. It is crowded, I find rest leaning against a wall next to a train door. I stare at the small screen in front of me, the number on the digital display slowly starts to rise, the train picks up speed, leaving the outskirts of the city to begin its travel through a sparsely populated landscape full of trees, greens, fields, small lakes and rivers, not much more, really. I look out the window, still tired from the restless sleep. Again and again we pass small places with their small houses, industrial buildings, sparsely developed infrastructure, as if from another time, familiar and almost forgotten. It seems that not much has changed here, but feels much more so where I had left 15 hours ago, now about 11,000 kilometres away, from a densely populated city-state very close to the equator. One house after another flashes by. Then silence again, accompanied by the monotonous rumblings of the moving train. Trees, fields, a sunny day, a good time to let yourself be carried away by fleeting fragments of thoughts while gazing into the distance.

\section{Spacecraft}

How far into space can I gaze from here? With the Hubble Space Telescope at one's hands, probably the farthest one can ever see is billions of light years away. The farthest we (or more precisely, a manmade machine) have travelled from earth is on Voyager 1, which left earth on September 5, 1977. A spacecraft now floats in interstellar space, a large-sized satellite dish with a diameter of 3.7 metres, equipped with an array of sensing devices to measure cosmic rays, electromagnetic fields and plasma waves. In 2018, as far from the Sun as from the Earth, it navigates in space guided by telemetry data and its Flight Data System, recording data on magnetic tapes. In 1990 and six billion kilometres away, it took a picture of home, our most distant view of Earth at barely the size of a pixel, also known as the Pale Blue Dot. Now at more than triple of the distance away, sending back data to Earth would take over 17 hours for a signal to reach after travelling for billions of kilometres through countless layers of space. The Voyagers will never make it back to Earth. Today the Falcon rockets go back and forth between space and Earth, deploying satellites, and at high precision they return and land on tiny platforms in the ocean. 


\section{Satellite}

I often miss the four seasons, time settles down in autumn. The cold, grey and short winter days I miss the least. Spring slowly turns winter from white to green, my parents have this beautiful garden that they care for day after day, spring is the time of year when nature's beauty begins to show itself. Summer is the weather I've gotten used to. The humidity doesn't bother me so much anymore, I found a good balance between the tropical heat and the cold breeze inside air-conditioned places often 10 degrees or more apart.

By chance I was able to photograph my parents' garden from a plane once. I could have easily looked it up online at a much higher resolution, even in $3 \mathrm{D}$, but certainly not as memorable as the photo I took on 30 June, 2017, just another day where over a thousand active satellites are orbiting Earth, sensing earthly processes, steadily collecting data, receiving and sending signals down to the ground. They studiously monitor environmental change, enable us to track and navigate our every step to show us the way. We don't see them, these invisible machines that operate the world we live in today. First launched in 1972 the Landsat satellites, these multispectral scanners capture photographic imagery of the Earth's land surface and coastal regions now for almost 50 years, followed by weather satellites, communication satellites, navigational satellites, anti-satellite weapons, and more. Picosatellites are emerging as DIY builder kits, Femtosats, at a wet mass between 10 to $100 \mathrm{~g}$, will be the smallest satellites coming out of university labs.

Many satellites are defunct but they still float in space, where else should they go? Dissolved into tiny pieces of junk at speeds of up to 28,000 kilometres per hour, they have become a growing danger to the orbit. Worst case scenario? Maybe the Kessler Syndrome, ${ }^{1}$ at a time where a new space race has just begun.

I am in a taxi now, on my way to town, the windscreen wipers move from left to right, right to left and wipe away a few drops of rain. A comforting song plays on the radio, probably a hit 30, 40 years ago, I am the "Eye in the Sky, looking at you ...." the meter reads $\$ 12.00$, the GPS on the mobile phone in front of the dashboard shows the way, trilateration $^{3}$ after trilateration.

I am the eye in the sky

Looking at you

I can read your mind

I am the maker of rules

Dealing with fools

I can cheat you blind

And I don't need to see any more to know that

I can read your mind, I can read your mind

- Eye in the Sky, The Alan Parsons Project.

\section{Unmanned Aerial Vehicle (UAV)}

At first it is an odd feeling to control the flight of an object just by looking through the lens of a camera. Attached to the remote control is a small screen, wirelessly connected to a white object hovering in midair. Unlike a phantom it is clearly visible, four rotors at a 90-degree angle
'The Kessler Syndrome refers to the collisional cascading of space debris. Collisions with debris create more debris and hence more collisions potentially threatening more objects in space like satellites and spacecrafts.

${ }^{2}$ Eye in the Sky is a song by rock band The Alan Parsons Project from their 1982 studio album of the same name. Speculations are that the song takes inspiration from George Orwell's book 1984 although satellites are not mentioned; others suggest that the 'eye in the sky' is a reference to the security cameras installed inside casinos monitoring gambling tables.

${ }^{3}$ As GPS satellites broadcast their location and time, trilaterations measure distances to pinpoint their exact position on Earth. Through the measurement of distances, your precise GPS location can be determined. https://gisgeography. com/trilateration-triangulation-gps. 
to each other, buzzing like a swarm of bees in full anticipation and at high frequency. A consumer product that lets you see the world from above, it is fun to fly these unmanned aerial objects (UAVs) which have now conquered the shelves of electronics shops: the phantoms, inspires, sparks or parrots. We not only see and hear about 'Big Brothers' in movies or over the news, but they are everywhere, they pervade, they are invisible like a ghost. Photos that are taken at incredulously high altitudes belie the size of these machines: they appear simple, minimalist in design, almost elegant, concealing their true power. Enclosed inside a sphere, mounted at the bottom of the flying object, sits a camera that can see sharper than the eyes of an eagle during the day or at night. Equipped with optical sensors good enough to read road signs from several kilometres away, capable of detecting, watching and tracking targets on command, it is operated often thousands of kilometres away with joysticks commonly used in video games.

Others like the Bramor Unmanned Aircraft Systems are well suited for surveying and remote sensing applications or where real-time, or near real-time, video observation and surveillance capabilities are needed. Optional enhancements include multi-spectral sensors for agriculture remote sensing, laser mass spectrometers for pipeline and environmental monitoring, or hyperspectral imagers. ${ }^{4}$ What started out as an art project, now operates as a company designing and manufacturing autonomous UAV systems out of Slovenia. In Rwanda, a zipline ${ }^{5}$ drone circles the sky, on a mission following its pre-programmed flight path, a lifesaving delivery service operating in remote rural areas, delivering urgent medicines and human blood conserves. On arrival at a destination it unlocks and drops its package, a red cardboard container with a parachute made of wax paper, slowly gliding down to the targeted base on the ground.

\section{Internet}

Remember Voyager 1 travelling in interstellar space? It is unimaginable in today's global communication systems for a signal to travel for hours, which is how long as it takes a signal from this spacecraft to reach Earth. At the equator it roughly takes 130 milliseconds for a signal to circumnavigate earth today and find its routes and destination inside our global nervous system - the internet. In the early 1960s J.C.R. Licklider's idea of a worldwide computer network sparked interest. With his colleagues at the Advanced Research Projects Agency he discussed the concept of the Intergalactic Computer Network which would later become an inspiration for the ARPANET (The Advanced Research Projects Agency Network), the basis for the internet as we know it today. The first signal sent on the internet was the letter L. Today, a day without internet is hardly imaginable, when everything is connected. Occasionally I come across an article where people go off the grid, disconnect from this rapidly built rhizomatic fabric, and I am almost tempted to join them. Otherwise we have to move on, continue to produce and consume information at our fingertips. Did you like Sophia's latest post? How many stars would you rate your trip today? Did you like the free sample we sent you? With every click and scroll we feed giant data centres with bits and pieces of our likes, thoughts, behaviours and moves. Cookies, super cookies - cute names for what has become a major tool amongst many others to track a user's habits, baked deeply into the roots of a browsing tool by nifty code, like trackable markers inside a specimen.
4 Technical details are taken from a list of sensors and optional enhancements on the C-Astral, http://www.c-astral.com.

5 The zipline drone is described in the article "Zipline: Help from above," by Jonathan W. Rosen in Best in Tech 2018, Special Edition, MIT Technology Review, p. 37. 


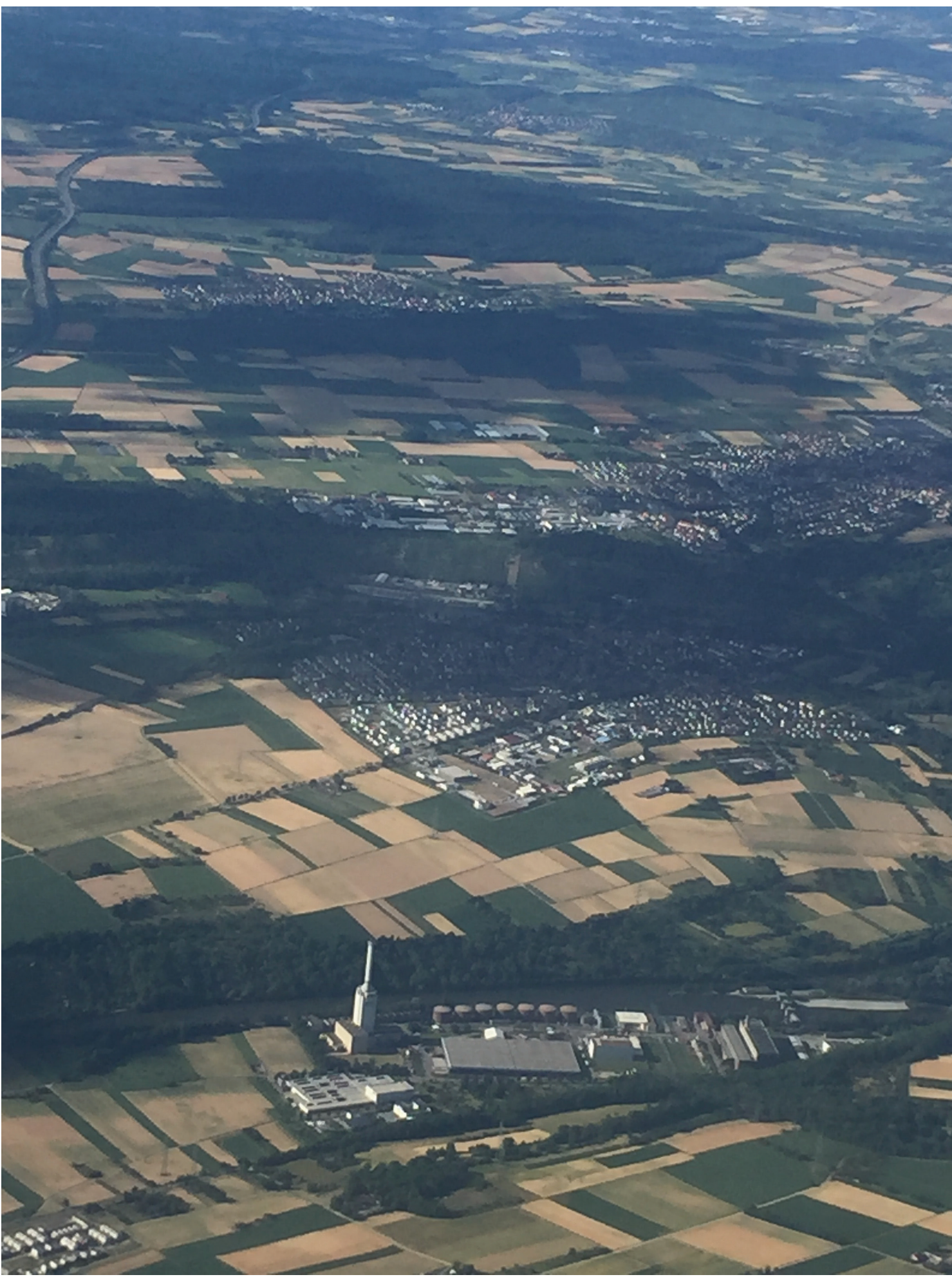

View taken with a phone while on a commercial flight, 30 June 2017 Photo: author 
It is not just us, it is the things, the Internet of Things, that gain much attention these days. Things that share their information with others, often sensors that detect events or changes in their environments: smart cities, smart homes, dark factories, connected machines, wearables, smart dust, the markers of specimen Earth. Cause and effect, infinite signals travelling across the planet at the speed of light. What do we do with trillions of connected things?

\section{City}

Cities are upgrading their infrastructures. Not necessarily just roads, buildings, public transportation, or recreational places, but infrastructures that we don't see, the omni-connecting optical fibres implanted into the ground, sensing devices woven into our architecture, bright LED lights. In the city of Wuhan that I have not heard of before I visited, the skyline at night is a must see, a synchronised masterpiece of countless networked light facades along the Yangtze river, stretching out into the far horizon. Cities are filled with Hertzian space, ${ }^{6}$ space that enables us to interact with our electronic devices, linking the immaterial with the material world, wireless networks, electromagnetic fields, radio signals, barely detectable by our senses, invisible to the human eye. New digital companions are moving in with us to assist us in our everyday lives, Alexa, Cortana, Azuma Hikari, Siri, although they are neither Samantha nor Joi yet.?

\section{Car}

Companies are competing over self-driving space, Uber, Tesla, Waymo, Apple. What started out as a grand challenge in the Mojave Desert is now taking over public streets. The one million dollar prize goes unclaimed during its first year in 2004. The farthest distance an autonomous car travelled then was measured at only 12 kilometres before it stalled on a rock. ${ }^{8}$ In the second year Stanley ${ }^{9}$ made it all the way to the finish line in under seven hours. Well equipped with state-of-the-art computer systems and sensors such as gyroscopes, accelerometers, GPS, LIDAR (LIght Detection and Ranging) and 100,000 lines of code, the robot car made its way across unknown terrain. LIDAR $^{10}$ sensors shoot millions of laser-light pulses into the air to generate a precise 3D representation of its surroundings as real as in a video game.

There is a manifold system of sensors built into a car today, a couple of distance sensors being one of them. Ever noticed a beeping sound when reversing your car? Beep, beep beep, beep beep beep. Stop. You just trust a distance sensor assisting you with parking your vehicle nicely into this tiny narrow parking spot. A self-driving car is equipped with at least eight, maybe 12 or more distance sensors. Trusting a self-driving car to safely get from A to B however, might still be a risk one has to take. Rocks are less of a concern, but finding solutions to problems such as the Trolley Problem ${ }^{11}$ or the Moravec's paradox ${ }^{12}$ are just mundane everyday situations that these self-driving cars face.

Imagine a runaway trolley is hurling down the tracks and is bound to hit either a group of five or a single person - would you kill one to save five?
${ }^{6}$ Hertzian Space is a term coined by Anthony Dunne and Fiona Raby that refers to the hidden electromagnetic environment generated by the increasing number of wireless devices.

7 Alexa, Cortana, Azuma Hikari, Siri are virtual assistants by Amazon, Microsoft, Gatebox, Apple respectively. Samantha and Joi are characters portraying virtual assistants in the movie Her and Blade Runner 2049 respectively.

${ }^{8}$ The DARPA grand challenge was a first-of-its-kind event and challenged the development of autonomous ground vehicles, http://archive. darpa.mil/grandchallengeo4/index. $\mathrm{htm}$

- Stanley was created by Stanford University's Stanford Racing team with the Volkswagen Electronics Research Laboratory (ERL)

10 LIDAR systems allow scientists and mapping professionals to examine both natural and manmade environments with accuracy, precision, and flexibility (https:// oceanservice.noaa.gov/facts/lidar. $h t m l)$ and is often used as a core technology in self-driving cars.

"The Trolley Problem is one of many ethical challenges the developments of self-driving cars are facing and is discussed in The everyday ethical challenges of self-driving cars, http://theconversation.com/theeveryday-ethical-challenges-of-selfdriving-cars-92710.

${ }^{12}$ Moravec's paradox is the discovery by artificial intelligence and robotics researchers Hans Moravec, Rodney Brooks and Marvin Minsky in the 1980 's that, contrary to traditional assumptions, high-level reasoning requires very little computation, but low-level sensorimotor skills require enormous computational resources. 
How would you spend your time in a self-driving car? Self-driving cars are usually not the bearers of good news these days. Most certainly this involves an accident and computational misfortunes.

Driver: Guys, the gear box is blocked. The gear box is blocked. Pitwall: Ok, working on it. Try to go to first.

Driver: I can't use rear.

Pitwall: Try reverse again, and then remove it and then try first again.

Driver: Something is broken, something is not working. ${ }^{13}$

The most sophisticated cars today are not made for public roads, but made for entertainment and innovation. Navigated by skilled drivers and remote control-centres, they rev their engines around circuits across the world, hunting for fractions of a second. I must admit I am a fan of these cars going in circles, restlessly chasing to beat the time sheets. Engines with almost a thousand horsepower, internal combustion machines, kinetic- and heat-motor generator units, turbochargers and arrays of sensors built into the Engine Control Unit, the Gear Control Unit, the Data logger Master Control Unit, the telemetry system, constantly connected to their race engineers, these aerodynamic masterpieces are results of endless computer simulations and wind tunnel tests-humans and machines on the edge.

\section{Robot}

TARS: Settings. General Settings. Security settings.

Cooper: Honesty, new setting: 95\%.

TARS: Confirmed. Additional customisation.

Cooper: Humour: $75 \%$.

TARS: Confirmed. Auto self-destruct T minus 10, 9.

Cooper: Let's make that $60 \%$.

TARS: $60 \%$ confirmed. Knock knock.

Cooper: You want $55 ?^{14}$

Absolute minimalist approach, Mies van der Rohe design, pure functional performance, it is just a block, the block is divided into smaller blocks, subdivided into smaller blocks, all with the same proportion as the original block. ${ }^{15}$ Robots often come in odd shapes, depictions of humans or animal like, but TARS and CASE from Interstellar are just blocks, beautiful simple blocks, elegantly moving and mastering every terrain. They are articulated planks with different characters, CASE is a softer but less experienced robot, TARS is developed to be this grizzled heartened middle-level officer, ex-marine.

SpotMini is advertised as a nimble robot, but unlike CASE and TARS, SpotMini is real. It has four legs, which makes it a quadruped, a longish body with synthetic intestines hidden under yellow armour. The multifunctional neck resembles a cross between a giraffe's neck and a robotic arm. Attached to the end of this arm is a mechanical hand that looks like a skeletal head. Cute or dreadful, one can't decide. This machine's value is only determined when being assigned a task. Others manoeuver difficult terrains or climb walls and trees with elegance and expertise. I am judging based on the demonstration videos I had watched.
${ }^{13}$ Excerpt from a radio conversation between a Formula 1 driver and his race engineer at the pitwall. After running wide, the car would not move back or forth.

\author{
${ }^{14}$ A dialogue from the 2014 movie \\ Interstellar between Joseph A. \\ Cooper, the pilot of the interstellar \\ expedition, and TARS, a tactical \\ robot, while Cooper repairs the \\ robot. \\ 15 Interstellar director and co-writer \\ Christopher Nolan elaborated on \\ the design of TARS and CASE in the \\ behind-the-scenes bonus interview \\ for the 2014 movie Interstellar.
}


A robotic arm is made up of several metal segments joined together. It is controlled by a computer system and rotated by individual motors. Its movement can be very elegant and precise, motion sensors feed back to the system to monitor its accuracy of movement. Over and over, it operates the same movement again and again, assembling and manufacturing one item after another. The factories these robots call their home are often in the dark and without air-conditioning, these machines don't need light to see or air to breathe.

What defines a robot? One robotic paradigm is Sense-Plan-Act: to gather data through sensors, create a world model from all this data, act upon it, then go back to step one. As such a robotic arm as much as a rice cooker qualifies as a robot. There is one thing robots can't do as well as humans though: pick strawberries. I've seen social robots - or humanoids or androids as they are often called - exhibited in museums. Their movements stutter easily and aren't as smooth as ours, their facial features and gestures still don't look quite right, like an adult doll looking at you in a curious but strange way, clearly distinguishable from a human being. When this is not true anymore you need tests, tests like the Voight-Kampff test.

You're in a desert walking along in the sand when all of the sudden you look down, and you see a tortoise, crawling toward you. You reach down, you flip the tortoise over on its back. The tortoise lays on its back, its belly baking in the hot sun, beating its legs trying to turn itself over, but it can't, not without your help. But you're not helping. Why is that? ${ }^{16}$

Ava is not real. She is anthropomorphic, sentient, a gynoid made of wires and futuristic materials instead of flesh, she is created and tested in an unknown place.

Ava: What will happen to me if I fail your test? Do you think I might be switched off because I don't function as well as I am supposed to?

Caleb: Ava, I don't know the answer to your question. It is not up to me.

Ava: Why is it up to anyone? Do you have people to test you that might switch you off?

Caleb: No.

Ava: Then why do I? ${ }^{17}$

\section{AI}

"I am sorry Dave, I am afraid I can't do that," HAL 9000 denies Dave reentry to the Discovery One spacecraft for fear that Dave will shut it down. HAL is not your ordinary computer, it is intelligent, it has the capacity to feel, perceive or experience subjectively, sentient-but HAL is also psychotic. ${ }^{18}$ HAL stands for Heuristically programmed ALgorithmic computer; others have suggested that HAL is a oneletter shift from IBM $=(\mathrm{H}+1)+(\mathrm{A}+1)+(\mathrm{L}+1)$. IBM, or International Business Machines, has built many computers with processing power doubling every two years, often the fastest and most powerful of their times. In 1997 IBM's Deep Blue, enclosed in a human-sized black box, beat world chess champion Gary Kasparow in a rematch $3 \frac{1}{2}-2 \frac{1}{2}$ by sheer processing speed and power, and good old-fashioned Artificial Intelligence. After the match Deep Blue was retired. IBM's Watson
${ }^{16}$ The Voight-Kampff is a fictional polygraph-like machine used by the LAPD's Blade Runners to assist in the testing of an individual to evaluate whether she/he is a replicant or not. http://bladerunner.wikia.com/wiki/ Voight-Kampff_machine.

\begin{abstract}
${ }^{17} \mathrm{~A}$ transcribed dialogue between gynoid Ava and young programmer Caleb Smith in the 2014 sci-fi fantasy movie Ex Machina.
\end{abstract}

${ }_{18}$ In 1966 Stanley Kubrick wrote a letter to Roger Caras, vice president of the film production company, asking if IBM was aware that HAL was a "psychotic computer". At the time IBM was one among other consultants for Kubrick's 1968 movie 2001: Space Odyssey. Letter correspondance http://www. lettersofnote.com/2013/o1/does-ibmknow-that-hal-is-psychotic.html. 
took on the challenge to win the game show Jeopardy! in 2011 to beat its opponents with its sheer processing speed and power, and Deep Content Analysis, Natural Language Processing (NLP), Information Retrieval (IR), Machine Learning (ML) and Artificial Intelligence (AI). A year later, Watson ventured into the challenges of cancer treatment. Once bedroom-sized, Watson now fits into a stack the size of three pizza boxes. In 2015 another machine went on a quest to beat the master of another game, the board game Go. AlphaGo ${ }^{19}$ the brainchild of Google DeepMind, is a computer program that, in 2016, defeated arguably the strongest Go player in history, Lee Sedol.

I thought AlphaGo was based on probability calculation and that it was merely a machine. But when I saw this move, I changed my mind. Surely AlphaGo is creative. This move was really creative and beautiful. This move made me think of Go in a new light. What does creativity mean in Go? It was really a meaningful move. ${ }^{20}$

What started way back then in 1956 when the Dartmouth Artificial Intelligence conference gave birth to AI, today, challenges the way we think about machines and intelligence.

In the 1950s and 1960s scientists built a few working perceptrons as these artificial brains were called. [..] This perceptron is being trained to recognise the difference between males and females it is something that all of us can do easily but few of us can explain how. To get a computer to do this would involve working out many complex rules about faces and writing a computer program. But this perceptron was simply given lots and lots of examples including some with unusual hairstyles. ${ }^{21}$

Machines attempt to make sense of their environment. Based on the data they have been trained on, their analysis and interpretation of their sensed environment is often not complete or fully accurate. They need to be fed better, they need to train harder, their gyms have to be better equipped. Often machines see the world differently than we do, even when they are doing countless tree searches in random forests:

... a tree trunk, a tree with no leaves, a tree trunk, a horse with a white hat, a white horse, a horse in the snow, trees with no leaves, a snow-covered hill, a black and white snowboard, a person riding a bike, a person on a snowboard, trees with no leaves, a person riding a bike, snow on the ground, a person is skiing, a tree with no leaves, a person holding a snowboard. ${ }^{22}$
19 AlphaGo is based on a deep neural network, a network of hardware and software, a technology that simulates neurons in the human brain. Version AlphaGo Lee would run on a distributed computer system. A later version, AlphaGo Zero, could be run on a single computer and beat the AlphaGo Lee 100:0.

${ }^{20}$ World Go champion Lee Sedol reflected on the $37^{\text {th }}$ move made by AlphaGo in the match's second game. It took more than 12 minutes of consideration before Sedol made his next move.

\footnotetext{
${ }^{21}$ Transcript of the generated captions by Youtube for the video clip Perceptron Research from the 50's \& 6o's, https:// www.youtube.com/watch?hl=en$\mathrm{GB} \& \mathrm{v}=\mathrm{cN}$ xadbrN_al.
}

\footnotetext{
${ }^{22}$ A transcript of a computer vision system detecting and identifying objects. The video shows a densely populated forest, the ground covered with snow, and a robot crossing the uneven terrain, in Random Forests, vimeo video $\sim 5: 46$, https://vimeo. com/244628946.
} 


\section{References}

C-Astral. http://www.c-astral.com. Accessed 3 June 2018.

"Does IBM know that HAL is psychotic?" Letters of Note. http://www. lettersofnote.com/2013/01/does-ibm-know-that-hal-is-psychotic.html. Accessed 25 June 208.

Ex Machina. Dir. Alex Garland. Perf. Domhnall Gleeson, Alicia Vikander, and Oscar Isaac. A24 and Universal Pictures, 2014. Film.

Grand DARPA Challenge. http://archive.darpa.mil/grandchallengeo4/ index.htm. Accessed 25 June 2018.

Himmelreich, Johannes. "The everyday ethical challenges of self-driving cars." The Conversation. https://theconversation.com/the-everydayethical-challenges-of-self-driving-cars-92710. Accessed 25 June 2018.

Interstellar. Dir. Christopher Nolan. Perf. Matthew McConaughey, Anne Hathaway, Jessica Chastain, Bill Irwin, Ellen Burstyn, and Michael Caine. Paramount Pictures and Warner Bros. Pictures, 2014. Film.

Perceptron Research from the 50's \& 6o's, clip. Uploaded by Pseudorntellectual on Youtube. https://www.youtube.com/watch?hl=en$\mathrm{GB} \& v=c N x a d b r N \_a l$. Video. Accessed 25 June 2018.

Random Forests. Uploaded by foam on Vimeo. https://vimeo. com/244628946. Video. Accessed 25 June 2018.

Rosen, W. Jonathan. "Zipline: Help from above." Best in Tech 2018, Special Edition. MIT Technology Review.

"Trilateration vs Triangulation - How GPS Receivers Work."

GISGeography. https://gisgeography.com/trilateration-triangulation-gps. Accessed 25 June 2018.

"Voight-Kampff machine." Fandom. http://bladerunner.wikia.com/wiki/ Voight-Kampff_machine. Accessed 25 June 2018.

"What is LIDAR?" National Ocean Service. https://oceanservice.noaa. gov/facts/lidar.html. Accessed 25 June 2018. 